\title{
COVID-19 Is Infecting Our Clinical Acumen
}

\author{
A. Jain, MD® and L. Chisick, MD, FRCPC \\ Department of Internal Medicine, University of Manitoba, Winnipeg, Manitoba, Canada.
}

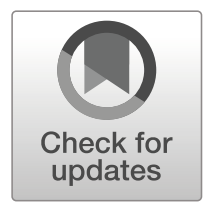

J Gen Intern Med 35(9):2750-1

DOI: $10.1007 / \mathrm{s} 11606-020-05997-7$

(C) Society of General Internal Medicine 2020

Y ou are standing in Mr. Smith's room, with two feet of space on either side of his hospital bed, trying to get a history from this ill 70-year-old man before you examine him. He's diaphoretic and appears septic. You see his scalenes working, desperately tugging at his ribcage to keep up with his respiratory effort. His vitals on the bedside display tell you his heart rate is ticking along at 130 beats per minute. His blood pressure is slightly lower than makes you comfortable. He coughs.

Suddenly, you are not thinking about the patient anymore. Is your PPE donned appropriately? Should you have worn an N95? Why did not you wear a scrub cap? It's getting harder for you to breathe under this surgical mask and you are starting to sweat under the gown. He coughs again.

You cut the interview short and tell the patient you are going to examine him. You grab the disposable stethoscope in the room and attempt to listen to his heart and lungs, turn his neck a few times, and with a fleeting poke at his abdomen and a peek at his feet, you rush out of the room.

You feel relieved to take your PPE off and you proceed with the ritual of scrubbing your hands until they feel raw. The only differential diagnosis that keeps coming to the forefront of your brain is COVID-19. For some reason, none of the other differential diagnoses seems important anymore - at least not until the COVID-19 swab result returns in a few days.

The fear surrounding COVID-19 is palpable, and the sense of insecurity and uncertainty has spread farther than the virus itself. At the Health Sciences Centre, the largest tertiary care centre in Manitoba, we spoke with several internal medicine residents and attending physicians at length about the effect of the pandemic on clinical medicine. There was consensus that the COVID-19 pandemic has affected the way in which they practice. The specialty known for its detail-oriented patient care and verbose progress notes seems to have forfeited its meticulous nature. Decreased time spent with patients, deferred in-depth physical examinations, clinic visits over

Received May 24, 2020

Accepted June 15, 2020

Published online June 30, 2020 the phone and a change in clinical acumen are examples of the ways in which medicine has been affected by this public health crisis.

In a recent survey conducted by Doctors Manitoba, 53\% of physicians said their health and wellness was a top concern, including the potential of contracting COVID-19 ${ }^{1}$. To highlight this point, several patient encounter notes now read "physical examination deferred," with listed reasons including "weighing the risk of exposure versus benefit." The fear and concern for our own safety, as health care workers, has taken a significant toll on the routine steps of patient interaction. A 2013 cohort study demonstrated that patients placed under routine contact precautions resulted in a $36 \%$ drop in visit frequency from health care workers. ${ }^{2}$ Extrapolating this in the context of a deadly pandemic virus makes it a fairly safe assumption that we are spending considerably less time with suspected or confirmed COVID-19 patients than other patients. These patients are then left even more susceptible to unintended adverse outcomes. ${ }^{2}$ Furthermore, we are discharging patients quicker than we would have in another era, despite the fact that they likely will not be seen in followup any time soon due to nonessential clinic closures. Perhaps this is to get them out of the incubators that tertiary care centres have become for COVID-19. Perhaps, it is to protect ourselves.

While internists have noticed their own clinical acumen affected, it is evident that physicians in other fields are experiencing similar issues. When the fears of COVID-19 first hit Manitoba, we were consulted on a cancer patient who had presented to the emergency department after waking up with sudden onset pleuritic chest pain and dyspnea. He was tachycardic and hypoxic upon arrival to the hospital. Bloodwork was unremarkable and the chest x-ray was clear. The patient was put on isolation precautions, swabbed for COVID-19, and given a dose of hydroxychloroquine. Approximately $12 \mathrm{~h}$ passed before another physician reassessed the patient and ordered a CT scan of his chest. The patient was subsequently found to have a massive pneumomediastinum, warranting an emergent thoracic surgery consult. Evident in this scenario, panic can cause the ordinarily skilled and seasoned physicians to have tunnel vision.

In addition, the pandemic has caused our morally and ethically driven thoughts to become somewhat dichotomized from our actions. Historically, when a hospitalized patient suffers a cardiac arrest, an assemblage of rescuers immediately swarms the bedside with the only objective being resuscitation 
of the patient. Now, in the age of COVID-19, an interim guidance published by the American Heart Association outlines several strategies that should be implemented prior to and during resuscitation of suspected and confirmed COVID-19 patients to protect health care workers from unnecessary exposure $^{3}$. Examples of these measures include ensuring all rescuers are in appropriate PPE prior to entry into the room, limiting personnel in the room, and even considering replacing manual chest compressions with mechanical CPR devices. Keeping this in mind, a delay as little as a few minutes in the initiation of CPR is associated with significantly decreased survival ${ }^{4}$. Although these measures are understandably in place to protect ourselves, we must recognize that they lead to poor outcomes for those that we are trying to help the most.

While the experience of the physician has certainly changed, it is the experience of the patient that has undoubtedly changed the most. We see patients at some of their most vulnerable points in their lives - when they are admitted to the hospital, having entrusted their health to a stranger. This stranger, the physician, quickly becomes a familiar face to trust. However, with COVID-19 imprisoning us in universal PPE, every face has morphed into the same eye shield, face mask, gloves, and gown. The empathy in our voices when telling a middle-aged man that he has stage IV colorectal cancer is now muffled by a mask, and our comforting body language is silenced by our protective gowns. Building a patient-doctor relationship has never been as difficult as it is today.

Despite the fears, risks, and rapidly evolving changes, we are trying our best to exercise the privilege we have to best serve our patients in this time of need. This is a time in our lives which we have never seen before and hope to never see again. It requires us to rapidly adapt to an evolving health care crisis, so it is no surprise that our clinical practice faces challenges as well. We are learning the safest way to both protect ourselves while protecting our patients, in order to ensure that we can continue to provide the best medical care as we work through this crisis together.

Corresponding Author: A. Jain, MD; Department of Internal Medicine, University of Manitoba, Winnipeg, Manitoba, Canada (e-mail: jaina3@myumanitoba.ca).

\section{Compliance with Ethical Standards:}

Conflict of Interest: The authors declare that they do not have a conflict of interest.

\section{REFERENCES}

1. COVID-19 Updates. Doctors Manitoba. 2020. URL: https:// doctorsmanitoba.ca/covid/covid19/. Accessed 29 April 2020.

2. Morgan DJ, Pineles L, Shardell $\mathbf{M}$ et al. The Effect of Contact Precautions on Healthcare Worker Activity in Acute Care Hospitals. Infect Control Hosp Epidemiol. 2013;34(1): 69-73. https://pubmed.ncbi.nlm.nih.gov/ 23221195/. Accessed 28 April 2020.

3. Edelson DP, Sasson C, Chan PS et al. Interim Guidance for Life Support for COVID-19. American Heart Association. 2020. https://www. ahajournals.org/doi/pdf/10.1161/CIRCULATIONAHA.120.047463. Accessed 29 April 2020.

4. Bircher NG, Chan PS, Xu Y et al. Delays in Cardiopulmonary Resuscitation, Defibrillation, and Epinephrine Administration all Decrease Survival in in-Hospital Cardiac Arrest. Anesthesiology. 2019;130;414-422. https://anesthesiology.pubs.asahq.org/article. aspx?articleid=2723487. Accessed 29 April 2020

Publisher's Note: Springer Nature remains neutral with regard to jurisdictional claims in published maps and institutional affiliations. 